\title{
Research and Application on Cogging effect of PMLSM
}

\author{
Yaohui $\mathrm{Li}^{1, \mathrm{a}^{*}}$ \\ ${ }^{1}$ College of Mechanical and Electrical Engineering of Xuchang University, Xuchang, PR China \\ aliyaohui@hust.edu.cn
}

Keywords: Cogging effect, Finite element analysis, Thrust fluctuation, PMLSM

Abstract. The purpose for research on cogging effect is to improve thrust fluctuation of PMLSM. As one of the main affecting factors of linear motor, its cogging effect is firstly researched and analyzed in this paper. And then we use finite element analysis software to perform the simulation so as to show that how cogging effect influences thrust of linear motor. Finally, it describes the influence degree and applicable range of cogging effect for the thrust fluctuation in contrast with Smooth armature model.

\section{Introduction}

As novel linear motion unit, high positioning accuracy and speed of PMLSM (permanent magnet linear synchronous motor) are come from its self-driven and no-intermediate-transmission-link structure。With the development of manufacturing, linear motor has become the main functional components of modern equipment. But its special structure generated byogging effect[1] has great influence on the output performance。Researching these disturbance factors and performing further optimization can make motor obtain good performance at a lower cost. In this paper, it shows that cogging effect have certain influence on permanent magnet motor performance by research of cogging effect, finite element analysis and the corresponding data comparing.

\section{Cogging effect of PMLSM}

Primary rotor slot can change the distribution of air gap magnetic field. Thus, it can also make magnetic intensity appear high harmonic component, which will result in the electric potential fluctuation and the motor runs extra loss. In addition, primary rotor slot can also directly cause the increase of the air gap reluctance, reduction of the effective flux and change of gas gap magnetic guide in movement. For a single slot, magnetic field model can be established according to the SchwarzChristoffel transformation equations and obtain the analytical results finally. However, it may be built on the basis of simplified model, and the calculation process and results are usually more complex.

Seeing from traditional permanent magnetic rotating motor, cogging effect of the linear motor and the magnetoresistance effect of traditional motor is consistent. Therefore, we can reference the analysis method of electric machine theory to qualitatively analyze PMLSM.

Schematic diagram of cogging effect is shown in Fig. 1. When it appears interaction between secondary permanent magnet and the primary magnetic field, the gap magnetic will change. The air gap magnetic at different positions is related with flux path with. In Fig.1 (a), the alveolar structure joining the air gap reluctance ensures $\lambda_{c 1}<\lambda_{c 2}$. And in Fig.1 (a), smooth armature structure can ensure that the air gap magnetic conductance is uniform, i.e., $\lambda_{s 1}=\lambda_{s 2}$ at any position.
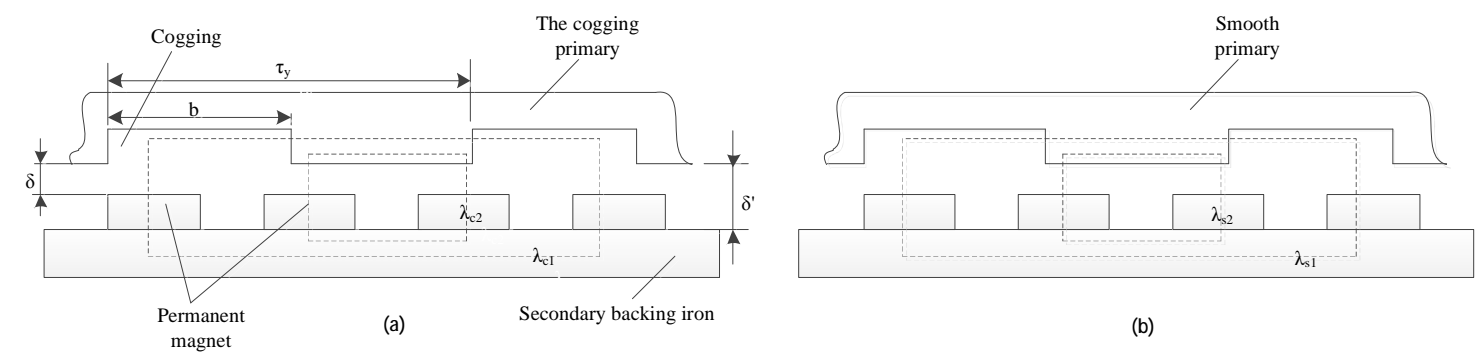

Fig.1 Schematic diagram of cogging effect 
The cogging will reduce the overall winding flux linkage. We usually increase the average magnetic flux and weaken cogging effect by introducing in the card's coefficient treatment $K_{c}$ in handling flux changes caused by cogging effect. The average magnetic flux density in the primary winding height is calculated by the smooth armature structure. In this case, reluctance relationship is met by $\lambda_{c 1}<\lambda_{s 1}=\lambda_{s 2}<\lambda_{c 2}$. Meanwhile, the air gap magnetic field model will be built by equivalent air gap height $\delta^{\prime}$, which will become function of permanent magnet and original air gap height. The model air gap height with the Chi's coefficient can be expressed as

$$
\delta_{e}=K_{c} \delta^{\prime}=K_{c}\left(\delta+\frac{h_{m}}{\mu_{r}}\right)
$$

where $K_{c}=1 /\left[1-\frac{b}{\tau_{y}}+\frac{4 \delta^{\prime}}{\pi \tau_{y}} \ln \left(1+\frac{\pi \tau_{y}}{4 \delta^{\prime}}\right)\right]$. The cogging force ripple is produced in a fixed cogging cycle distribution. It can be expressed by the Fourier equation

$$
F_{s}(x)=p F_{d n}(x)=p \sum_{n=1}^{\infty} a_{n} \sin \left(n \frac{2 \pi x}{\tau}\right),
$$

where $F_{d n}(x)$ is magnetic resistance of a single magnetic pole, $p$ is number of pole pairs. Therefore, the total cogging force is sum of cogging force separately generated by every permanent magnet, i.e.,

$$
F_{a s}(x)=\sum_{p=1}^{m} \sum_{n=1}^{\infty} b_{n} \sin \left(n \frac{2 \pi x}{\tau}+\varphi_{n}\right)
$$

where, $m$ is the number of permanent magnet, $b_{n}$ is cogging force generated by the $\mathrm{p}$-th permanent magnet, $\varphi_{n}$ is the initial phase angle.

For the analysis of the cogging effect, the mathematical analysis results are often obtained by simplified solution. And it is difficult to establish a model for solving the problem. Therefore, we often use finite element method to analyze the cogging effect.

\section{Finite element analysis of cogging effect}

Firstly, parameters, sign and corresponding values of linear motor can be shown as follow:

(1) In primary armature, it mainly includes: the number current phase (m)-3; the number of poles (p)-6; number of turns per phase (N)-240; copping number (Q)-18; cogging pitch (t)-12mm; cogging width (ts)-6mm; cogging depth (ds): $30 \mathrm{~mm}$; height of primary iron core (a): $50 \mathrm{~mm}$; length of iron core (1): $222 \mathrm{~mm}$.

(2) In secondary permanent magnet, it includes: polar distance $(\tau)-36 \mathrm{~mm}$; permanent magnet height (h)-3mm; permanent magnet width(w)-30mm.

(3) Air gap height (g)-1mm.

(4) In winding, it includes electric current (I): $2 \mathrm{~A}$; resistance $(\mathrm{R}): 5 \Omega$.

Cogging effect is mainly caused by the force fluctuation, which is generated by the change of air gap magnetic conduction in the slot. The analysis direction is how the cogging structure parameters influences force fluctuation[2]. Another direction is how the external factors influence cogging effect.

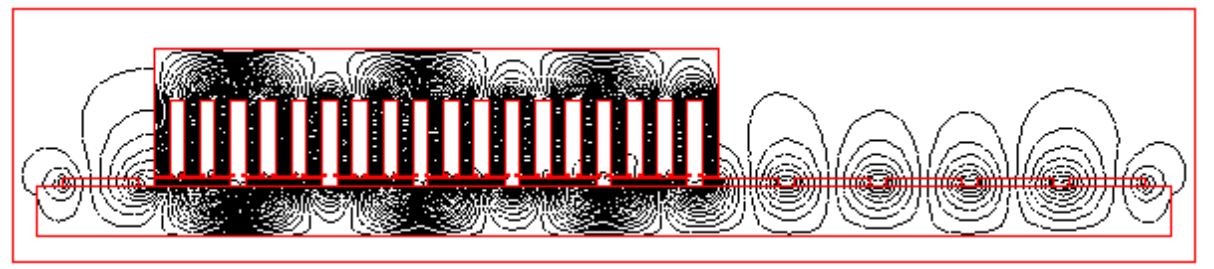

Fig. 2 The cogging model of PMLSM with no power

When it exists cogging and power is disconnected in PMLSM, its magnetic field distribution can be shown in Fig. 2. It is obvious that the distribution of the magnetic field has a bigger change in 
contrast with magnetic field distribution of smooth armature because the magnetic resistance is Invisibly increased under the condition that we assume the magnetic permeability of the coil and the magnetic conductance of the air are both 1 . In this case, it will eventually affect the change of magnetic resistance. According to the Maxwell tensor method, computational results on magnetic field thrust are: $F_{x}=650.7 N \quad F_{y}=46695 N$.

When the three-phase current is accessed to the primary winding of a linear motor, electric coil will generate traveling wave magnetic field, which can interact with permanent magnetic field of permanent magnet to accumulate energy in the air gap and generate electromagnetic force promoting the primary mover. In this case, the linear motor will have driving load capacity itself.

According to the theory of motor and the PMLSM parameters model, The process of the windings' arrangement is as follows: cogging number of each pole and each phase $q=\frac{Z}{2 p m}=\frac{18}{2 \times 3 \times 3}=1, \alpha_{1}=\frac{p \times 360^{\circ}}{18}=60^{\circ}$. According to the EMF star graph of $60^{\circ}$ phase belt, it should include six phase belts, i.e., A, B, C, X, Y, Z, which use the single-layer-winding way, and the number of parallel branch $\mathrm{a}=1$, and then arrangement of three phase winding can be shown in Fig. 3 .

The magnetic field distribution is shown in Fig. 4 after the three-phase current is accessed. Results on magnetic field thrust are: $F x=2769 N, F y=55533 N$.

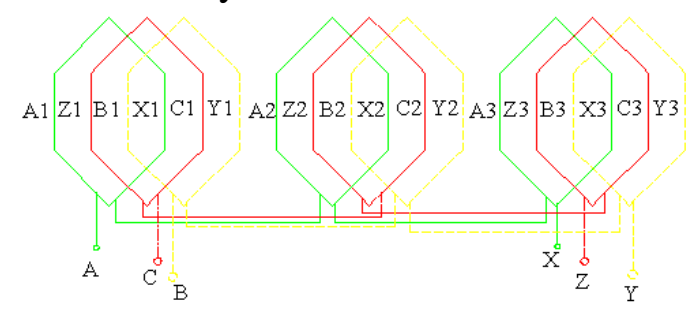

Fig. 3 Mode of connection for single layer winding

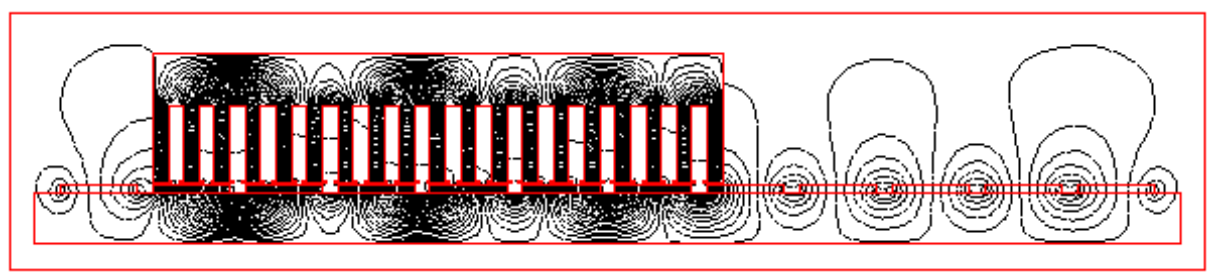

Fig. 4 The cogging model of PMLSM with power

The primary structure parameters (such as length, cogging parameters) of PMLSM, with multi-variable parameters and high-coupling characteristic, will have a big impact on its performance. There is no direct relationship (their sizes) between the cogging existence and the beginning of the end, therefore, we can handle them separately. In view of this idea, force wave of cogging motor model can be equivalent to the superposition of end effect and cogging force.

The force data model shown in Fig. 5 is built by the following two conditions: 1) smooth armature model and cogging armature model are both use the step distance $\mathrm{s}=1 \mathrm{~mm} ; 2$ ) their moving distance ( $24 \mathrm{~mm})$ is twice as long as tooth distance. In addition, they have the same initial position, where 'ef' is the end effect force curves obtained under the smooth armature model, '(e+t)f' is the force curve of cogging model. According to the processing method of superposition, result for ' $(e+t) f$ ' subtracting 'ef' is 'tf', which is equivalent to single cogging force. We can conclude that the fluctuation of cogging force is with tooth distance $t$ as cycle. The analysis result is consistent with reality.

Furthermore, normal attractive force distribution is shown in Fig. 6. Due to the presence of cogging, it reduces the action range of the magnetic field. Therefore, the normal attractive force of smooth armature is much more than that of cogging armature. But the normal attractive force of cogging armature is more than that of smooth armature in the range of twice tooth distance. The main reason is the existence of cogging, it will make the magnetic field distribution of the secondary permanent magnetic field become more uniform, increase the number of high harmonics[3, 4] and have bigger fluctuation. 


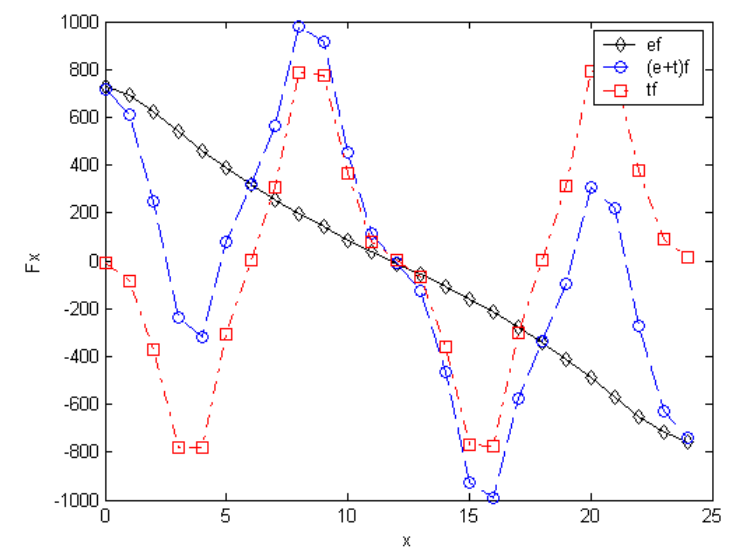

Fig. 5 Comparison result of end effect force and cogging force

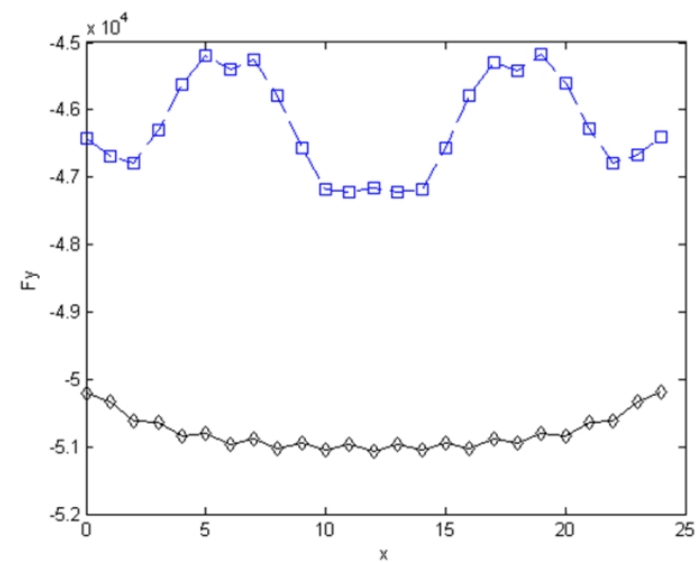

Fig. 6 Normal force comparison resulst on smooth and cogging armature

\section{Summary}

By analyzing and researching, we can draw the following conclusion: (1) the fluctuation of cogging force is with tooth distance $t$ as cycle; (2) the finite element analysis result is basically coincide with reality; (3) Cogging effect can improve force fluctuations in a certain application range; (2) it can also offer a new idea for structure optimization of linear motor.

\section{Acknowledgement}

This work is supported by the Henan Natural Science Foundation (No. 14A460023).

\section{References}

[1] Luo, Y., et al., Fractional order robust control for cogging effect compensation in PMSM position servo systems: stability analysis and experiments. Control Engineering Practice, 2010. 18(9): p. 1022-1036.

[2] Luo, Y., Y. Chen, and Y. Pi. Authentic simulation studies of periodic adaptive learning compensation of cogging effect in PMSM position servo system. in Control and Decision Conference, 2008. CCDC 2008. Chinese. 2008. IEEE.

[3] Luo, Y., Y. Chen, and Y. Pi, Cogging effect minimization in PMSM position servo system using dual high-order periodic adaptive learning compensation. ISA transactions, 2010. 49(4): p. 479-488.

[4] Luo, Y., et al. A high order periodic adaptive learning compensator for cogging effect in PMSM position servo system. in Systems, Man and Cybernetics, 2008. SMC 2008. IEEE International Conference on. 2008. IEEE. 